\title{
A critical appraisal of the quality of guidelines for radiation protection in interventional radiology using the AGREE II tool: a EuroAIM initiative
}

A quality assessment of radioprotection guidelines in interventional radiology

\section{Abstract}

Purpose: To systematically review and assess the methodological quality of guidelines for radiation protection in interventional radiology.

Materials and Methods: On April 15 ${ }^{\text {th }}, 2021$, a systematic search for guidelines on radiation protection in interventional radiology was performed using MEDLINE, EMBASE, National Guideline Clearinghouse, and National Institute for Health and Clinical Excellence databases. Among retrieved guidelines, we then excluded those not primarily focused on radiation protection or on interventional radiology. Authors' professional role and year of publication were recorded for each included guideline. Guideline quality evaluation was performed independently by three authors using the six-domain tool "AGREE II", with an overall guideline quality score divided into three classes: low $(<60 \%)$, acceptable $(60 \%-80 \%)$, and good quality $(>80 \%)$.

Results: Our literature search identified 106 citations: after applying exclusion criteria, 11 guidelines published between 2009 and 2018 were included, most of their authors being interventional radiologists $(168 / 224,75 \%)$. Overall quality of included guidelines was acceptable (median $72 \%$, interquartile range $64 \%-83 \%$ ), with only one guideline (9\%) with overall low quality and four guidelines (36\%) with overall good quality. Among AGREE II domains, "Scope and Purpose", "Clarity of Presentations", and "Editorial Independence" had the best results (87\%, 76\%, and 75\% respectively), while 
"Applicability", "Rigor of Development", and "Stakeholder Involvement" the worst (46\%, 49\%, and 52\% respectively).

Conclusion: Considering all guidelines, the overall methodological quality was acceptable with one third of them reaching the highest score class. Domain "Applicability" had the lowest median score, highlighting a practical implementation gap to be addressed by future guidelines.

Keywords (MeSH terms): Patient safety; Interventional radiology; Radiation protection; Dosimetry; Systematic review; Guideline; AGREE II

\author{
Abbreviations: \\ AGREE: Appraisal of Guidelines for Research \& Evaluation \\ EuroAIM: European Network for the Assessment of Imaging in Medicine \\ ICRP: International Commission on Radiological Protection \\ IQR: interquartile range
}




\section{Introduction}

While clinical benefits of interventional radiology are widely recognized and outweigh the potential harms of the related use of ionizing radiation, the increase in the number of fluoroscopically guided procedures implies substantial radiation exposure risks both for patients and staff [1]. Professionals involved in interventional procedures are subjected to lower radiation exposure than patients, mainly due to exposure to lower-energy scattered radiation and to the relatively higher distance from the primary radiation beam in comparison to patients [2]. However, the total radiation dose these professionals receive throughout their career can be high enough to become a matter of concern.

In this view, the International Commission on Radiological Protection (ICRP) and the European Directive on Basic Safety Standards include staff radiological risk as a variable to be considered both in the justification and in the optimization of radiation exposure for medical purposes [3]. However, the responsibilities of the referrer and of the practitioner go far beyond reasonable exposure justification and optimization. The lack of standardized radiation safety measures and of specific radiation protection training for professionals working with fluoroscopy, especially outside radiology departments [4], could heighten radiation exposure and strengthen its harmful effects. Such risks renewed the attention on a widespread awareness for radiation safety [5], intensifying the application of radiation protection strategies $[6,7]$.

Several clinical practice guidelines issued by major medical societies or by specialized working groups have summarized the established knowledge on radiation protection, in order to systematize and improve the safety of interventional and diagnostic procedures. However, since these efforts were not coordinated in a general framework, the methodological quality of those publications is affected by an 
unavoidable variability [8,9]. In this scenario, an extensive appraisal of available guidelines is a relevant issue. As in other clinical settings [10], these appraisals are conducted by systematically applying quality evaluation tools, such as the Appraisal of Guidelines for Research \& Evaluation (AGREE) tool - updated in 2009 with the AGREE II tool - that is reported to be a reliable, validated, and internationally used standard for the critical assessment of guidelines [11,12]. In the diagnostic and interventional imaging field, such evaluations have been recently advocated and carried out [13-17] by the European Network for the Assessment of Imaging in Medicine (EuroAIM), a working group of the European Institute for Biomedical Imaging Research [18].

To the best of our knowledge, such kind of evaluation has never been conducted on guidelines for radiation protection in interventional radiology. Thus, we aimed to critically appraise the recently updated evidence-based guidelines on this topic, using the AGREE II quality assessment tool. 


\section{Materials and methods}

\section{Search strategy and eligibility criteria}

No ethics committee approval was required for this systematic review. On April $15^{\text {th }}$, 2021, a systematic literature search targeting practice guidelines for patient safety in interventional radiology was performed using MEDLINE (PubMed; www.pubmed.gov), EMBASE (Embase; www.embase.com), Google (Google; www.google.com), National Guideline Clearinghouse (www.guideline.gov; key words: 'interventional radiology' and 'safety'), and the National Institute for Health and Clinical Excellence (www.nice.org.uk; key words: ‘interventional radiology’ and 'safety') databases. A controlled vocabulary (medical subject headings in PubMed and EMBASE thesaurus keywords in EMBASE) was used. Keywords included "guidelines" OR "practice guidelines" AND “interventional radiology" AND "safety", and their expansions. No limits were applied to the literature search timeframe.

The search key was: ('practice guideline'/exp OR 'clinical practice guidelines' OR 'guidelines' OR 'guidelines as topic' OR 'practice guideline' OR 'practice guidelines' OR 'practice guidelines as topic' OR 'consensus development'/exp OR 'consensus development' OR 'consensus development conference' OR 'consensus development conferences' OR 'consensus development conferences as topic' OR 'consensus development conferences, nih' OR 'consensus development conferences, nih as topic') AND ('interventional radiology'/exp OR 'interventional radiography' OR 'interventional radiology' OR 'radiography, interventional' OR 'radiology, interventional' OR 'surgical radiology' OR 'vascular and interventional radiology' OR 'fluoroscopy'/exp OR 'fluorescence radiation' OR 'fluorescence scanning' OR 'fluorescent scanning' OR 'fluorophotography' OR 'fluoroscopy' OR 'photofluoroscopy' OR 'radiofluoroscopy' OR 
'roentgen fluoroscopy' OR 'roentgenfluoroscopy' OR 'scopy, fluoro' OR 'x ray fluoroscopy' OR 'xray fluoroscopy') AND ('patient safety'/exp OR 'patient safety') AND ('radiation dose'/exp OR 'dose rate, radiation' OR 'dose, radiation' OR 'radiation dosage' OR 'radiation dose' OR 'radiation dose absorption' OR 'radiation dose output' OR 'radiational dose' OR 'radiobiological dose' OR 'radiological dose' OR 'radiation'/exp OR 'multiple field radiation' OR 'non ionizing radiation' OR 'nonionizing radiation' OR 'radiation' OR 'radiation change' OR 'radiation control' OR 'radiation incidence' OR 'radiation quality' OR 'radiation, nonionizing').

We excluded from the results of our search those guidelines that were not primarily focused on patient safety in interventional radiology, such as national/international guidelines in which radiation dose management was only briefly mentioned in the context of a more comprehensive safety evaluation. In case of updated guidelines, we always considered the most recent version, also ultimately excluding: posters, oral presentations, animal studies, review articles, guidelines not issued by national and international medical societies (e.g., designed for local use), and articles with full manuscript unavailable in English. The initial selection of eligible articles was performed by two independent readers (BLINDED and BLINDED, with 3-4 years of experience in the evaluation of practice guidelines, respectively) considering titles and abstracts only. After downloading eligible articles, the full texts were reviewed, and references were manually searched for further eligible guidelines.

\section{Guideline evaluation}

The evaluation of guidelines quality was performed using the AGREE II tool, through the official online platform [19]. The AGREE II tool (Table 1) consist of 23 different 
items organized in 6 domains: domain 1 (items 1-3) called "Scope and Purpose"; domain 2 (items 4-6) "Stakeholder Involvement”; domain 3 (items 7-14) "Rigor of Development"; domain 4 (items 15-17) "Clarity of presentation"; domain 5 (items 1821) “Applicability”; domain 6 (items 21-22) "Editorial Independence”. These six domains are followed by two additional items (“Overall Assessment"), which include "the rating of the overall quality of the guideline and whether the guideline would be recommended for use in practice".

Three researchers (BLINDED, BLINDED, and BLINDED), with 1-3 years of experience in the use of the AGREE II tool, independently appraised each included guideline. Each of the AGREE II items and the two global rating items are rated on a 7point scale from 1-(strongly disagree) to 7 (strongly agree). According to the AGREE II User Manual, a score of 1 (strongly disagree) was given if there was no available information relevant to the AGREE II item, or if the concept was very poorly reported. Conversely, a score of 7 (strongly agree) was given if the quality of reporting was optimal and complete. Scores between 2 and 6 were assigned when the reporting of the AGREE II item did not meet the full criteria or considerations, and represent a progressive rate of partial completeness. Upon completing the 23 items, taking into account all criteria considered in the assessment process, all reviewers provided an overall evaluation of the guideline to recommend its practical usage, with three available options: "Yes", "Yes, with modifications", and "No". Another author (BLINDED, with 4 years of experience in the use of the AGREE II tool) then calculated standardized domain scores for each of the six domains, as recommended by the AGREE II checklist [19], according to the following formula:

standardized score $=\frac{(\text { obtained score }- \text { minimum possible score })}{(\text { maximum possible score }- \text { minimum possible score })} \times 100 \%$ 
For each eligible article we also recorded authors' professional roles, year of publication, geographic origin of the research group, presence of a multidisciplinary panel, and eventual involvement of patient advocacy groups.

\section{Data synthesis}

We used descriptive statistics to summarize the characteristics of guidelines deemed eligible for inclusion. Raw and aggregated data were summarized as frequency (percentage) or median and interquartile range (IQR). As mentioned above, for each guideline, we calculated a quality score for each of the six domains, using the formula presented in the AGREE II User's Manual [19]. Domains were considered as of "Low" quality when the overall quality score was lower than $60 \%$, of "Acceptable" quality when the overall quality score was between $60 \%$ and $80 \%$, and of "Good" quality when the overall quality score was higher than $80 \%$. 


\section{Results}

\section{Search results}

Our systematic search resulted in 106 citations, while 3 further articles were retrieved from website and index searches. After the first study selection, based on abstract or article type, 56 articles and related documents underwent full-text screening, 11 of them - published between 2009 and 2018 - ultimately meeting inclusion criteria [20-30]. Fig. 1 depicts the flowchart of the guideline selection process.

Table 2 summarizes the total score for each domain as well as the overall quality judgment. Of the eleven evaluated guidelines, all except one [25] reached an acceptable quality level $(\geq 60 \%)$ in overall evaluation, four of them $[20,21,23,26]$ attaining the highest quality score $(\geq 80 \%)$. No guideline however reached an acceptable score in all six domains: the guideline by Dauer et al. [26] had an insufficient score in Domain 5 ("Applicability"), while the guideline by Stecker et al. [20] had an insufficient score in Domain 2 (“Stakeholder Involvement").

Acceptability in Domain 1 ("Scope and Purpose") was obtained by all guidelines, with scores ranging from $70 \%$ [25,27] to $98 \%$ [21]. Domain 1 was also the best in terms of overall results considering all evaluated guidelines (median 87\%, IQR 83\%-94\%). Guideline quality was low both in Domain 2 ("Stakeholder Involvement") with a median score of 52\% (IQR 44\%-57\%) ranging from 31\% [20] to 69\% [26], and in Domain 3 ("Rigor of Development"), with a median score of 49\% (IQR 37\%-64\%). Domain 4 ("Clarity of Presentation") had a median quality score of 76\% (IQR 63\%79\%), while Domain 5 (“Applicability”) had the lowest median score (46\%, IQR 44\%54\%), ranging from 33\% [27] to 63\% [25]. Finally, Domain 6 ("Editorial Independence") reached an intermediate median quality score of 75\% (IQR 54\%-89\%). 
Median quality at overall assessment was 72\% (IQR 64\%-83\%), 6/11 (55\%) guidelines being assigned a practical use recommendation of "Yes, with modification", $5 / 11(45 \%)$ an unconditional "Yes" recommendation for practical use.

When evaluating the subspecialty of the authors of each included guideline, we observed how the majority of authors were interventional radiologists $(168 / 224,75 \%)$, followed by medical physicists $(34 / 224,15 \%)$, and other professionals such as diagnostic radiologists, cardiologists, pediatricians, epidemiologists, and nurses $(22 / 224,10 \%)$. No trends were observed considering the publication year and the median overall quality assessment. 


\section{Discussion}

We reported the quality appraisal of the most recent guidelines on patient safety and radiation protection in interventional radiology, conducted using the AGREE II tool. A key finding of our evaluation, ultimately including 11 guidelines as a result of a systematic review, was the highly variable quality across all six AGREE II domains: the Domain 1 (Scope and Purpose) obtained the highest overall assessment score (87\%), whereas the Domain 5 (Applicability) obtained the lowest one (46\%).

The high compliance (87\%) of included guidelines to Domain 1 (Scope and Purpose), focused on the overall aim of the guideline, the clinical question, and the target population), could be mainly due to their strict focus on patient safety and radiation protection, which is relevant in all interventional radiologic procedures. Conversely, widespread lack of involvement of patients' associations and advocacy groups resulted in a low score in Domain 2 (Stakeholder Involvement) which specifically targets the degree to which a guideline represents the views of all involved subjects. An equally unsystematic reporting both of methodological information (such as evidence search methods and selection criteria) and of details on the recommendation formulation process (such as description of considered health risks, benefits, and side effects) resulted in an insufficient evaluation for Domain 3 (Rigor of Development) in $6 / 11(55 \%)$ of the included guidelines. Of note, only $2 / 11(18 \%)$ guidelines reported a procedure for their own update. Domain 4 (Clarity of Presentation) reflects the adequacy in the reporting of recommendations and different options for radiation dose management, being unsatisfactory in only $2 / 11$ guidelines $(18 \%)$ : key recommendations were easily identifiable, specific, and unambiguous, as requested by the AGREE II Tool. Domain 5 (Applicability) was the lowest scoring domain, reporting results similar 
to other articles [17], while Domain 6 (Editorial Independence) showed good quality in most of the guidelines, except for the reporting of competing interests by members of the guideline development groups.

Our analysis of guidelines on radiation protection in interventional radiology showed that the most critical issue is their applicability in the daily routine. The low score of the Applicability domain mirrors the difficulties that professionals in the interventional suite encounter even when applying simple practical advices, such as those reported in the 2010 CIRSE guideline [21]. For example, the recommendation for the operator to stand on the side of the transmitted beam when using lateral angulated projections frequently collides with procedural timings. Another example of a poorly applied recommendation could be the correct and constant use of effective collimation, that is frequently disregarded due to the need of having a broader view of the operating field and to avoid to lose possible areas of interest. In this case, specific training provided to operators should highlight how proper x-ray beam collimation to the area of interest improves image quality and also curtails patient and operator dose by reducing scattered radiation. However, this scenario is also influenced by limitations in technological equipment and staff composition, since the increasingly complex procedures performed in the interventional suite require highly-coordinated multidisciplinary teams. Even to comprehensively implement the key points for safe practice proposed by the 2010 CIRSE guideline [21], that would aid to reduce or minimise occupational radiation dose, a second operator with specific knowledge and skills on radiation dose optimisation would be required. This second operator would be in charge of ensuring adequate dose monitoring, collimation, tube-detector distance, positioning, use of protective shielding. Unfortunately, while all these tasks could be 
committed to radiographers, who are specifically trained to operate medical imaging equipment in a manner that optimizes image quality and minimizes patient and clinical personnel exposure [31], diffuse availability of such professionals to act as second operators in this capacity widely varies among European countries [32-35].

Another point that must be addressed is that only six on eleven guidelines included in this review were published after the release of the 2013 EURATOM directive on radiation protection [36]. Future guideline updates will therefore have to take into account how countries have implemented the EURATOM directive into their national regulations, in particular article number 60 , which states that any equipment used for interventional radiology must have a device or a feature that, during and after the procedure, can inform all professionals carrying out practical aspects of the procedures about the amount of radiation produced by the equipment. Indeed, while accurate monitoring of occupational radiation dose limits in the angiographic suite is paramount to ensure staff safety, only in the CIRSE [21] and Jones et al. [29] guidelines we found indications regarding the correct wearing of dosimeters. This highlights a potential gap in the evaluation of staff radiation exposure during X-ray-guided procedures, resulting in inaccurate (or even incorrect) monitoring of operator doses $[37,38]$. Of note, recent technological advances, such as active electronic personal dosimeters, have been proven to effectively optimize radiation dose monitoring even at department-wide level $[39,40]$. These dosimeters, wirelessly linked to a central database, allow processing dose data for each operator and for each procedure, under the control of centralized dose management systems [39,40]. In developing new guidelines or updating existing ones, authors should take into consideration these new tools, not only for active monitoring but also to provide operators with real-time alerts 
that allow to discriminate between high doses derived from complex procedures and high doses caused by improper use of protective measures. This would increase operator awareness and engagement, resulting in day-by-day training towards a dose reduction attitude.

Finally, guideline applicability could highly benefit from a comprehensive involvement of all professionals in charge of radiation protection in the guideline drafting process. This extension needs to consider not only the professionals formally responsible for radiation protection, such as physicians and medical physicists, but also radiographers, nurses and all practitioners involved in the field of interventional radiology. This advice appears more and more relevant when considering the ever increasing clinical role of interventional radiology.

Some limitations of our assessment should be considered. First, the AGREE II tool does not assess the consistency of guidelines with corresponding published evidence and does not evaluate the clinical content of guidelines. Moreover, selection of guidelines was uncertain, since the definition of guidelines is not universally established, and we observed a concentration of 9/11 (82\%) guidelines in the same journal (Journal of Vascular and Interventional Radiology, Elsevier, USA), a relatively expected result considering the prominent role of this journal in the field of interventional radiology in the last twenty years. However, we cannot exclude that some guideline might have been missed by our strategy search.

In conclusion, our analysis revealed high quality guidelines concerning radioprotection in interventional radiology only in $36 \%$ the assessed guidelines. The Applicability domain was the lowest in terms of overall score, highlighting a general lack of guidance for the routine implementation of these recommendations, with an 
equally fragmented presentation of guideline monitoring and auditing criteria.

Considering these findings, we first advise that professionals who already consult and apply the current guidelines should try to draw the most robust recommendations from each guideline. Then, we recommend that future guidelines should have a much stronger focus on prompt and clear applicability, from both a technical and a clinical point of view. Improved applicability could be best achieved by a more extensive involvement of all healthcare professionals dealing with interventional procedures, addressing risks and attaining radiation exposure minimization by using appropriate safety measures. 


\section{Author contributions:}

BLINDED: Conceptualization, Methodology, Writing - Original Draft, Writing Review \& Editing, Visualization, Supervision. BLINDED: Formal Analysis, Writing Original Draft, Writing - Review \& Editing. BLINDED: Formal Analysis, Writing Original Draft, Writing - Review \& Editing, Visualization. BLINDED: Formal Analysis, Writing - Review \& Editing. BLINDED: Formal Analysis, Writing - Review \& Editing. BLINDED: Formal Analysis, Writing - Review \& Editing. BLINDED: Formal Analysis, Writing - Review \& Editing. BLINDED: Formal analysis, Data Curation, Writing - Review \& Editing, Visualization. BLINDED: Methodology, Investigation, Data Curation, Writing - Review \& Editing. BLINDED:

Conceptualization, Methodology, Supervision, Writing - Review \& Editing, Project Administration.

Funding: The authors state that this work has not received any funding.

Conflict of Interest: BLINDED declare that they have no conflict of interest and that they have nothing to disclose.

BLINDED declares to be member of speakers' bureau/advisory board for BLINDED. BLINDED declares to have no conflict of interest and declares to have received grants from or to be member of speakers' bureau/advisory board for BLINDED, BLINDED, and BLINDED. 


\section{References}

[1] Panuccio G, Greenberg RK, Wunderle K, Mastracci TM, Eagleton MG, Davros W. Comparison of indirect radiation dose estimates with directly measured radiation dose for patients and operators during complex endovascular procedures. J Vasc Surg 2011;885-894.e1. doi: 10.1016/j.jvs.2010.10.106

[2] Miller DL, Balter S, Cole PE, et al. Radiation Doses in Interventional Radiology Procedures: The RAD-IR Study Part I: Overall Measures of Dose. J Vasc Interv Radiol 2003;711-727. doi: 10.1097/01.RVI.0000079980.80153.4B

[3] Bartal G, Vano E, Paulo G, Miller DL. Management of Patient and Staff Radiation Dose in Interventional Radiology: Current Concepts. Cardiovasc Intervent Radiol 2014;289-298. doi: 10.1007/s00270-013-0685-0

[4] European Society of Radiology (ESR). Summary of the European Directive 2013/59/Euratom: essentials for health professionals in radiology. Insights Imaging 2015;411-417. doi: 10.1007/s13244-015-0410-4

[5] Gopee-Ramanan P, Reis S. Principles of Radiation Safety in Interventional Radiology. Demystifying Interv Radiol 2016. p. 9-13. doi: 10.1007/978-3-31917238-5_3

[6] Adamus R, Loose R, Wucherer M, Uder M, Galster M. Strahlenschutz in der interventionellen Radiologie. Radiologe 2016;275-281. doi: 10.1007/s00117-0160083-0

[7] Degiorgio S, Gerasia R, Liotta F, et al. Radiation Doses to Operators in Hepatobiliary Interventional Procedures. Cardiovasc Intervent Radiol 2018;772_780. doi: 10.1007/s00270-017-1870-3

[8] Kung J, Miller RR, Mackowiak PA. Failure of Clinical Practice Guidelines to 
Meet Institute of Medicine Standards. Arch Intern Med 2012;1628. doi: 10.1001/2013.jamainternmed.56

[9] Shaneyfelt T. In Guidelines We Cannot Trust. Arch Intern Med 2012;1633. doi: 10.1001/2013.jamainternmed.335

[10] Makarski J, Brouwers MC. The AGREE Enterprise: a decade of advancing clinical practice guidelines. Implement Sci 2014;103. doi: 10.1186/s 13012-0140103-2

[11] Brouwers MC, Kho ME, Browman GP, et al. AGREE II: advancing guideline development, reporting and evaluation in health care. Can Med Assoc J 2010;E839-E842. doi: 10.1503/cmaj.090449

[12] Brouwers MC, Kerkvliet K, Spithoff K. The AGREE Reporting Checklist: a tool to improve reporting of clinical practice guidelines. BMJ 2016;i1152. doi: 10.1136/bmj.i1152

[13] Messina C, Bignotti B, Bazzocchi A, et al. A critical appraisal of the quality of adult dual-energy X-ray absorptiometry guidelines in osteoporosis using the AGREE II tool: An EuroAIM initiative. Insights Imaging 2017;311-317. doi: $10.1007 / \mathrm{s} 13244-017-0553-6$

[14] Romeo V, Stanzione A, Cocozza S, et al. A critical appraisal of the quality of head and neck cancer imaging guidelines using the AGREE II tool: A EuroAIM initiative. Cancer Med 2019;209-215. doi: 10.1002/cam4.1933

[15] Messina C, Bignotti B, Tagliafico A, et al. A critical appraisal of the quality of adult musculoskeletal ultrasound guidelines using the AGREE II tool: an EuroAIM initiative. Insights Imaging 2017;491-497. doi: 10.1007/s13244-0170563-4 
[16] Romeo V, Stanzione A, Ugga L, et al. A Critical Appraisal of the Quality of Glioma Imaging Guidelines Using the AGREE II Tool: A EuroAIM Initiative. Front Oncol 2019;472. doi: 10.3389/fonc.2019.00472

[17] Doniselli FM, Zanardo M, Manfrè L, et al. A critical appraisal of the quality of low back pain practice guidelines using the AGREE II tool and comparison with previous evaluations: a EuroAIM initiative. Eur Spine J 2018;2781-2790. doi: $10.1007 / \mathrm{s} 00586-018-5763-1$

[18] Sardanelli F, Bashir H, Berzaczy D, et al. The Role of Imaging Specialists as Authors of Systematic Reviews on Diagnostic and Interventional Imaging and Its Impact on Scientific Quality: Report from the EuroAIM Evidence-based Radiology Working Group. Radiology 2014;533-540. doi:

10.1148/radiol.14131730

[19] My AGREE PLUS | AGREE Enterprise website. Available via https://www.agreetrust.org/resource-centre/agree-plus/ Accesse July 312021

[20] Stecker MS, Balter S, Towbin RB, et al. Guidelines for Patient Radiation Dose Management. J Vasc Interv Radiol 2009;S263-S273. doi:

10.1016/j.jvir.2009.04.037

[21] Miller DL, Vañó E, Bartal G, et al. Occupational Radiation Protection in Interventional Radiology: A Joint Guideline of the Cardiovascular and Interventional Radiology Society of Europe and the Society of Interventional Radiology. Cardiovasc Intervent Radiol 2010;230-239. doi: 10.1007/s00270-0099756-7

[22] Baskin KM, Hogan MJ, Sidhu MK, et al. Developing a Clinical Pediatric Interventional Practice: A Joint Clinical Practice Guideline from the Society of 
Interventional Radiology and the Society for Pediatric Radiology. J Vasc Interv Radiol 2011;1647-1655. doi: 10.1016/j.jvir.2011.07.010

[23] Dauer LT, Thornton RH, Miller DL, et al. Radiation Management for Interventions Using Fluoroscopic or Computed Tomographic Guidance during Pregnancy: A Joint Guideline of the Society of Interventional Radiology and the Cardiovascular and Interventional Radiological Society of Europe with Endorse. J Vasc Interv Radiol 2012;19-32. doi: 10.1016/j.jvir.2011.09.007

[24] Miller DL, Balter S, Dixon RG, et al. Quality Improvement Guidelines for Recording Patient Radiation Dose in the Medical Record for Fluoroscopically Guided Procedures. J Vasc Interv Radiol 2012;11-18. doi:

10.1016/j.jvir.2011.09.004

[25] American College of Radiology, Society of Interventional Radiology, Society of Neurointerventional Surgery, Society of Pediatric Radiology. Practice Parameter for Interventional Clinical Practice and Management. J Vasc Interv Radiol 2015;1197-1204. doi: 10.1016/j.jvir.2015.05.017

[26] Dauer LT, Miller DL, Schueler B, et al. Occupational Radiation Protection of Pregnant or Potentially Pregnant Workers in IR: A Joint Guideline of the Society of Interventional Radiology and the Cardiovascular and Interventional Radiological Society of Europe. J Vasc Interv Radiol 2015;171-181. doi: 10.1016/j.jvir.2014.11.026

[27] Rafiei P, Walser EM, Duncan JR, et al. Society of Interventional Radiology IR Pre-Procedure Patient Safety Checklist by the Safety and Health Committee. J Vasc Interv Radiol 2016;695-699. doi: 10.1016/j.jvir.2016.03.002

[28] Baerlocher MO, Kennedy SA, Ward TJ, et al. Society of Interventional Radiology 
Position Statement: Staffing Guidelines for the Interventional Radiology Suite. J Vasc Interv Radiol 2016;618-622. doi: 10.1016/j.jvir.2016.02.010

[29] Jones AK, Dixon RG, Collins JD, Walser EM, Nikolic B. Best Practice Guidelines for CT-Guided Interventional Procedures. J Vasc Interv Radiol 2018;518-519. doi: 10.1016/j.jvir.2017.10.021

[30] Hirshfeld JW, Ferrari VA, Bengel FM, et al. 2018 ACC/HRS/NASCI/SCAI/SCCT Expert Consensus Document on Optimal Use of Ionizing Radiation in Cardiovascular Imaging-Best Practices for Safety and Effectiveness, Part 1: Radiation Physics and Radiation Biology. Catheter Cardiovasc Interv 2018;203-221. doi: 10.1002/ccd.27660

[31] European Federation of Radiographer Societies (EFRS). European Qualifications Framework (EQF) Level 6 Benchmarking Document: Radiographers. Available via https://api.efrs.eu/api/assets/publications/139 Accessed 31 July 2021

[32] Pasieka E, Lewandowski J, Żuk J. The role and responsibilities of a radiographer in a cardiac cath-lab. Journal of Public Health, Nursing and Medical Rescue No.1/2014 (11-14). Available via http://pzpr.eu/numery/2014_1/201413.pdf Accessed 31 July 2021

[33] Lundén M, Lundgren SM, Lepp M. The nurse radiographers' experience of meeting with patients during interventional radiology. Journal of Radiology Nursing 2012;31:53-61. doi: 10.1016/j.jradnu.2011.11.001

[34] Anderson J, Zanardo M, Smyth B, Fox L, Anderson A, Maher M, Rainford L. An interventional cardiology investigation: patient exposure to radiation and interoperator variability in an Irish setting. Radiation Protection Dosimetry. 2020;192(89-96). doi: 10.1093/rpd/ncaa201 
[35] Schegerer AA, Frija G, Paulo G, et al. Radiation dose and diagnostic reference levels for four interventional radiology procedures: results of the prospective European multicenter survey EUCLID. Eur Radiol. 2021. doi: 10.1007/s00330021-08029-y.

[36] European Council Directive 2013/59/Euratom of 5 December 2013 laying down basic safety standards for protection against the dangers arising from exposure to ionising radiation, and repealing Directives 89/618/Euratom, 90/641/Euratom, 96/29/Euratom, 97/43/Euratom and 2003/122/Euratom. Available via https://eurlex.europa.eu/eli/dir/2013/59/oj Accesse 31 July 2021

[37] Lopez P O, Dauer L T, Loose R et al. 2018 ICRP Publication 139: Occupational radiological protection in interventional procedures. Ann ICRP; 47:1-118

[38] Gerasia R, Ligresti D, Cipolletta F, et al. 2019 Endoscopist's occupational dose evaluation related to correct wearing of dosimeter during X-ray-guided procedures. Endosc Int Open. 2019 Mar; 7(3):E367-E371. doi: 10.1055/a-08413350.

[39] Vano E, Sanchez Casanueva RM, Fernandez Soto JM, Bartal G. 2021 Challenges in Occupational Dosimetry for Interventional Radiologists. Cardiovasc Intervent Radiol. doi: 10.1007/s00270-020-02725-w.

[40] Vano E, Sanchez Casanueva RM, Fernández Soto JM. Helping to know if you are properly protected while working in interventional cardiology. J Radiol Prot. 2020. doi: 10.1088/1361-6498/abc325. 


\section{Figure and table legends}

Fig. 1 Flowchart for the study selection and exclusion process.

Table 1 AGREE II domains description and item synthesis.

Table 2 Total score obtained by all guidelines for each domain. Domain 1: "Scope and Purpose"; Domain 2, "Stakeholder Involvement"; Domain 3, "Rigor of Development"; Domain 4, "Clarity of Presentation"; Domain 5, “Applicability”; Domain 6, "Editorial Independence”. 